\title{
APROXIMACIÓN A LOS ENSAMBLES ENTRE EXCLUSIÓN, PRODUCCION Y TERRITORIO
}

\author{
Carlos Fidel \\ Raúl Di Tomaso \\ Cristina Farias ${ }^{* * *}$
}

\begin{abstract}
Abordar la problemática productiva cruzada con los aspectos socioeconómicos, localizados en el territorio de la Zona Metropolitana de la Ciudad de Buenos Aires (ZMCBA).

Metodología: El recorrido metodológico transita por la presentación contextual, se continúa en el sendero analítico, en términos territoriales y temáticos. El lenguaje se despliega en torno a dos conceptos centrales: Producción y Pobreza. Ambas nociones abren amplias y distintas líneas de reflexiones e interpretaciones, muchas de ellas más o menos confrontadas. Presentaremos una perspectiva de los dos "conceptos" y examinaremos los posibles enlaces que se pueden establecer entre ellos. Finaliza con una serie de propuestas que pueden servir de disparador de nuevos trabajos o de posibles acciones concretas para mejorar la situación de vida los sectores más pobres.

PALAVRAS-CHAVE: produção, pobreza, exclusão, qualidade de vida, territorio.
\end{abstract}

\section{INTRODUCCIÓN}

El presente trabajo se encuentra organizado en tres bloques temáticos. Primero se hace referencia a los antecedentes macroeconómicos de la Argentina en las últimas décadas. Del mismo modo, y como derivado del contexto anterior, se realiza una síntesis del contenido y de los efectos de las políticas sociales durante el mismo período. A continuación se presenta una síntesis histórica del concepto de capital social y su aplicación en el mundo social, para luego realizar algunas reflexiones críticas al concepto, con el objetivo de sumergirse en las bases teóricas sobre las que se construyen los modelos rígidos y homogéneos de las políticas sociales dirigidas a "combatir" la pobreza en América Latina.

* Doutor em Economia. Professor titular da Faculdade de Ciências Sociais da Universidad Nacional de Quilmes - UNQ. R. S. Peña 352 (B1876BXD) Buenos Aires, Argentina. chfidel@unq.edu.ar

* * Sociólogo pela Universidad de Buenos Aires - UBA - e Mestre pela UNTREF. Professor e pesquisador do Observatorio PyME.rdtomaso@unq.edu.ar

*** Posgraduada em Economía Urbana. Professora e pesquisadora da UNQ. cfarias@unq.edu.ar
Luego se inicia una línea temática de investigación, avanzando sobre el análisis de la situación de pobreza en un recorte territorial delimitado ubicado al sur del Área Metropolitana de Buenos Aires (AMBA), utilizando, para ello, los datos oficiales que solo pueden dar cuenta de una faceta, aquella que se genera a partir de la falta de ingreso - Línea de Pobreza (LP) y Línea de Indigencia (LI) -, y las necesidades básicas insatisfechas (NBI), dando cuenta de una visión economicista, fragmentada y desintegrada de un fenómeno complejo como es la pobreza. A pesar de ello, esta información nos permite incorporar el análisis del sistema productivo local del territorio estudiado, basándonos en las pequeñas y medianas empresas, con el objetivo de mostrar la vulnerabilidad en la estructura y funcionamiento del sector y sus limitaciones en la generación de puestos de trabajo más estables, que puedan contribuir a mejorar la situación social de los sectores más desposeídos de esta comunidad en particular.

Para finalizar, se presentan las conclusiones emanadas del cruce de los dos temas anteriores y se elabora una serie de recomendaciones de políticas 
económicas y sociales tendientes a contribuir en la solución de las penosas condiciones de vida que atraviesan amplios sectores de la población del lugar

\section{PRINCIPALES SUCESOS PRECEDENTES}

\section{Antecedentes contextuales}

A fines de 2001, cuando en la Argentina se produjo el abrupto desplome del denominado Plan de Convertibilidad, se exhibió de manera descarnada y virulenta el nuevo mapa social, económico y político que, a partir de fines de la década del setenta, se fue delineando con la aplicación de políticas económicas y sociales de pleno corte neoliberal.

El diseño del Plan de convertibilidad, que comenzó a aplicarse en 1991, tenia como eje central, en el trazado interno, generar las bases para fundar una plena estabilidad de las variables monetarias de largo plazo. Este objetivo se sustentaba en la creación de una unidad monetaria directamente ligada al comportamiento del dólar norteamericano; conformado de tal modo, significaba un instrumento de política monetaria rígido, que acotaba la cantidad de unidades monetarias que circulaba internamente a la medida disponible de reservas en divisas externas. Dicho instrumento limitaba el comportamiento del gasto público a la disponibilidad de moneda extranjera.

A la dimensión anterior se sumaba un eje de política externa que buscaba abrir íntegramente la economía argentina al mercado mundial, con el objetivo procurar la modernización de los procesos de producción y consumo, adoptando las nuevas tecnologías de base digital en la comunicación y organización de los circuitos de producción y circulación de los bienes y servicios.

\section{Los efectos sociales de las políticas públicas aplicadas en los años noventa}

La morfología socioeconómica que se fue configurando en la década del noventa se reflejó en un alto nivel de concentración de la riqueza en segmentos más reducidos de la población, en la desconcentración y el achicamiento de la incidencia del aparato y las funciones estatales, la desconexión del funcionamiento del sistema bancario y financiero del comportamiento productivo, el desacoplamiento de los eslabones productivos internos y la intensa transnacionalización empresarial de los circuitos productivos, de servicios y financieros.

El tipo de cambio establecido, en esos años, promovió las importaciones y obstaculizó las exportaciones, especialmente las de origen industrial. En consecuencia, se fue configurando un fuerte proceso de "desindustrialización”, en tanto que el perfil económico, utilizando las ventajas fijas y naturales, se fue especializando en ampliar la frontera productiva del sector primario y en el desarrollo de los procesos para transformar esa producción.

La ampliación del crédito hipotecario orientó a un segmento significativo de las nuevas inversiones hacia el sector de la construcción y el mercado inmobiliario, lo cual amplió la oferta de bienes de ese tipo de los segmentos más caros y dio lugar a nuevos productos en zonas cerradas en área suburbanas o en complejos habitacionales con sofisticados satisfactores internos al terreno.

Este considerable cambio de la producción y el consumo fue acompañado por una descomunal expansión de la segregación social y territorial; una de las principales consecuencias, en la dimensión territorial, especialmente en el uso, la distribución y apropiación del espacio urbano, fue que tendió a profundizar la segmentación socioespacial y constructiva. También se registró el aumento de las relaciones laborales informales y exclusión social, que afectó sobre todo a los sectores históricamente más vulnerables, sumando así, de manera creciente, nuevos afectados por la brutal reducción de las posibilidades concretas de insertarse en el mercado laboral.

Incorporado al anterior escenario, es de señalar el descreimiento y descomposición de toda forma orgánica y representativa del funcionamiento 
de distintas instituciones políticas, culturales y, en menor medida, de las de carácter religioso y educativas.

\section{EL DESARROLLO CONCEPTUAL DE “CAPI- TAL" Y SU APLICACIÓN EN EL MUNDO SOCIAL}

El concepto de "capital social" se remonta a principios del siglo xx, en el marco de la pedagogía. Luego, en los años cincuenta, es retomado por los estudios económicos relacionados con el desarrollo de las economías (Fidel; Di Tomaso; Farías, 2008, p. 22).

Robert Solow fue uno de los especialistas que investigó el tema con la intención de definir, en términos conceptuales y en el sentido clásico, aquellos factores intangibles que obstaculizaban a las sociedades deshacerse de situaciones que impedían su desarrollo económico y social. En sus inicios, el concepto de capital social se focalizaba en las dimensiones tecnológicas de los procesos productivos y actuaba como una variable condicionante del desarrollo de los países.

A mediados del siglo pasado, la mayor capacidad tecnológica estaba agrupada en un núcleo concentrado de países con un desarrollo industrial relativo. Se trataba de explicar los diferentes grados de desarrollo por la capacidad tecnológica diferencial entre ellos, comparando dos modelos de organización de la economía: Por un lado, aquella que se sustentaba sobre los mecanismos de funcionamiento del mercado y, por otro, una economía apoyada sobre un modelo totalmente opuesto, donde el mercado era sustituido por la intervención de un Estado centralizado, que planificaba toda la actividad económica.

Dentro de este contexto internacional, Latinoamérica presentaba un panorama heterogéneo de desarrollo industrial, con algunos enclaves industriales promovidos por la aplicación de políticas públicas, pero que no lograban expandirse al resto del territorio y solo en algunos pocos casos evolucionaban favorablemente y crecían.

El concepto de capital social tomó un nuevo impulso a partir de la década de los ochenta, en los estudios especializados en temas de política social. Autores como Bernardo Kliksberg, retomaron el concepto que luego adoptaron los organismos multilaterales de crédito y la Comisión Económica para América Latina y el Caribe CEPAL.

El nuevo sentido adoptado por el concepto se incorporó rápidamente en las investigaciones de las ciencias sociales, que le confieren múltiples interpretaciones y tratan de dar cuenta de las formas de vida social y de las condiciones de pobreza de la población. En síntesis, se trataba de cuantificar estos activos derivados de las relaciones sociales.

Este enfoque nos lleva reflexionar en torno a algunos conceptos: capital y social. ¿Son dos categorías compatibles? ¿Cuáles son los factores que miden el uso de estas categorías? ¿Poseen alguna utilidad en los resultados que producen?

\section{UNA APROXIMACIÓN CRÍTICA AL CONCEPTO DE CAPITAL SOCIAL}

Un aspecto significativo es la ambigüedad con
que se alude y utiliza el término de "capital soci-
al”. Se lo emplea para definir varios campos ana-
líticos y, en muchos casos, se lo confunde con las
energías de la productividad de la fuerza de
trabajo, mientras que en otros se entiende como
el grado y la calidad de capacitación que tienen
los trabajadores. (Fidel; Di Tomaso; Farías, 2008,
p. 23).

Este criterio, adoptado por organismos multinacionales, se toma con el objetivo de identificar las distintas redes o lazos sociales que se dan entre los diferentes segmentos del tejido social, principalmente entre los sectores más pobres de la población y que incluyen, en muchos de los casos, a las vinculaciones entre cuestiones étnicas, de género, religiosas, ideológicas y políticas, entre otras.

Esta corrienterealiza su abordaje metodológico suponiendo una organización social ideal, localizada en un territorio urbano o rural determinado, con ausencia de conflicto social, donde los pobladores actúan de manera solidaria, honesta, 
egoísta, sin intereses particulares o corporativos, para aumentar la competitividad colectiva y obtener beneficios económicos en un mercado amplio, abierto y social.

Dentro de esta comunidad imaginada, la ausencia de conflictos está idealizada y, si estos surgieran, pueden ser solucionados de manera sencilla, pero al parecer existen obstáculos internos y externos en estas comunidades, que frenan de algún modo el desarrollo social y el acceso al capital.

No está definido claramente cómo operan estas trabas dentro de las sociedades, pero se supone que, si es visible el diseño de la estructura del capital social, la estrategia superadora del conflicto se dará con el aporte de las comunidades de buena voluntad y predisposición para superarlo. Entonces, las acciones de una política pública o privada deben estar dirigidas a consolidar y desarrollar, en el ámbito de una sociedad local, el "capital social" que se encuentra en la esencia de toda comunidad de manera latente, y solo hay que dejarlo aflorar y difundirlo.

En su mayoría, el diseño y la estrategia de aplicación de estas acciones son elaborados desde el exterior de las comunidades, y son universalmente aplicables en todo espacio urbano o rural y en cualquier momento en el tiempo. Pueden verse resultados favorables en un corto período y con notables mejoras materiales y simbólicas.

Los expertos en el diseño de las propuestas de intervención, si se revelan resultados no favorables o de logros poco sustantivos, argumentan, ante estos actos fallidos, la carencia de recursos, fallas en la aplicación de la propuesta, por no seguir al pie de la letra los manuales elaborados por especialistas, o fallas en ciertos factores y actores, entre otros motivos. En este sentido, los expertos que adoptan este enfoque consideran que las ideas son buenas, pero existen grietas que impiden su correcta aplicación, y, por esas razones, los pobres no alcanzan a capturar el capital.

Aunque sea social, es un capital que se evapora en los recorridos de los actores encargados de aplicar las recetas y en los receptores que se resisten a formar parte de los beneficios magnánimos del capital, patrimonio social que se puede extender a través de los mecanismos de los espacios del mercado, que es amplio, generoso y receptivo a la difusión del capital. (Fidel; Di Tomaso; Farías, 2008, p. 24).

Por último, cabe señalar que sintéticamente se han analizado y planteado los rasgos básicos de estas estrategias operativas, que conforman el discurso que transita por los senderos de aplicación de las políticas sociales en América Latina, con recetas homogéneas que no contemplan las especificidades de las diferentes comunidades locales, zonas y países.

En este sentido, es importante destacar que las estrategias, en el horizonte de los organismos públicos y privados, nacionales y multinacionales, están fuertemente arraigadas, y se advierten pocas señales de brisas renovadoras de cambio en el pensamiento instalado, con ánimo de iniciar la búsqueda y el montaje de un nuevo paradigma superador de análisis, que permita diseñar innovadoras estrategias operativas para abordar definitivamente las penosas dificultades que sufren grandes sectores de la población.

\section{INICIOS DEL NUEVO SIGLO CON EL FINAL DE UN MODELO}

La crisis que, a fines de 2001, se expresó en la desarticulación social, institucional, económica y financiera en la Argentina tuvo efectos múltiples. A nivel de las respuestas sociales se registraron:

- acentuación de las búsquedas de salidas individuales, en muchos casos, optando por los senderos informales o al margen de la legalidad; - profundización y desarrollo de los lazos sociales y la creación o ampliación de nuevos movimientos basados en organizaciones de índole asociativa y solidaria.

En esta última dimensión, hay que marcar el surgimiento de nuevas modalidades de movimientos sociales. Señalaremos los principales: uno de ellos estaba motivado por los afectados por el sistema bancario, mientras que otro era de carácter territorial urbano, diferenciando las dis- 
tintas corrientes de los movimientos de desocupados (piqueteros), algunos inaugurando nuevas modalidades de construcción del hábitat o formas productivas. También emergieron nuevas modalidades asociativas en torno a asambleas barriales, fenómeno que se registró especialmente en la Ciudad de Buenos Aires. Algunas de estas formas organizativas de base popular fueron efímeras, mientras que otras resisten el paso del tiempo.

Desde el Estado, se adoptaron respuestas tardías y poco imaginativas dentro de los esquemas de políticas gubernamentales "tradicionales". La estrategia gubernamental se desplazaba como si la crisis fuera una recesión más o un pasaje transitorio por una catastrófica situación inevitable que, cada cierto período, atraviesa al país.

Pasados los momentos más agudos de la crisis, desde el Gobierno se aplicó una política cambiaria que fortaleció el perfil primario exportador y la sustitución de bienes y servicios externos; ello generó a que poco a poco se recomponga el mercado interno y se registren altas tasas de crecimiento de la producción; además, se intentó, con resultados positivos, acordar una salida para restablecer los flujos de créditos externos con los organismos multilaterales de crédito y reconstituir el funcionamiento de la esfera bancaria y financiera.

$\mathrm{Al}$ mismo tiempo, en el plano social, se empezó a distribuir un "donativo" de dinero en efectivo destinado a la población más expuesta a la crisis. La administración y gestión de los pequeños montos asignados a los responsables de las familias expulsadas del mercado laboral fue resuelta unilateralmente desde la esfera del Estado. Dicha instrumentación estuvo marcada por procedimientos pocos transparentes y sin una activa participación de los actores sociales involucrados.

\section{LOS ASPECTOS SOCIALES EN LA ZONA SUR DEL AMBA}

El recorte territorial que analizaremos en este artículo se encuentra ubicado al sur del Área Metropolitana de la Ciudad Autónoma de Buenos
Aires, y está conformada por nueve municipios que ocupan una superficie total de $820,9 \mathrm{~km}^{2}$. Se trata de un área densamente poblada, e involucra a casi tres millones de habitantes. Durante la última década, la tasa de crecimiento poblacional ha presentado una desaceleración en algunas zonas del territorio, que se corresponden con los asentamientos de los sectores de mayores ingresos, quizá porque la región ha perdido la capacidad de atraer migraciones internas, debido al cambio y decaimiento en su capacidad generadora de empleo, como la dada entre los años sesenta y setenta, y las condiciones macroeconómicas desfavorables a nivel de la economía en su conjunto. En el otro extremo, se posicionan las porciones de territorio donde se ubican los asentamientos de los barrios más pobres en los diferentes municipios, en los cuales se observaron importantes tasas de crecimiento y, en muchos casos, las cifras entre los períodos de censos (19912001) ascendieron al $60 \%$, una tendencia que se destaca en los municipios ubicados más al sur en el territorio de estudio.

Si bien es cierto que las estadísticas oficiales disponibles para analizar los temas propuestos presentan, a nuestro criterio, ciertas falencias metodológicas que no permiten captar los diferentes matices y carecen de indicadores con capacidad de dar cuenta de la complejidad del fenómeno, a los fines de este trabajo poseen un cierto grado de utilidad para mostrar que existe una vinculación entre la pobreza y el sistema productivo, sobre todo en lo que se refiere al papel fundamental que desarrollan las pequeñas y medianas empresas en la generación de empleo. Un trabajo futuro deberá incursionar en establecer e investigar los ensambles existentes, o por crear, entre pobreza y sistema productivo local.

Los indicadores oficiales están centrados en diferenciar los distintos niveles de riqueza y pobreza de la población, dando lugar a los términos "línea de pobreza" e "indigencia”, este último referido a la población más pobre entre los pobres.

El eje analítico, empleado en métodos muéstrales de sostén empírico, transita por cuantificar el 
ingreso que recibe la población; paralelamente se confecciona una canasta de consumo necesaria para que una familia tipo pueda reproducirse, y se determina cuál es el ingreso necesario para comprar los productos comprendidos en dicha canasta. Atravesando el nivel de ingreso con el precio de la canasta de bienes básicos, deviene el gradiente de acceso al consumo de la población (Fidel; Di Tomaso; Farías, 2008, p. 33.).

La concepción de pobreza por ingresos que captan los datos oficiales utilizados giran en torno a la idea de la incapacidad de los hogares de adquirir una canasta de bienes y servicios, reduciendo el concepto de pobreza a la incapacidad de consumo, estableciendo una brecha entre pobres y no pobres, de necesidades básicas insatisfechas, y teniendo, en su concepción, una visión economicista, fragmentada y desintegrada de la pobreza que se contrapone a las argumentaciones y definiciones clásicas en torno al tema de numerosos autores que han definido a la pobreza como un fenómeno complejo, multifacético y relacional. Estos autores (Senn, Arriagada, Barba, Ziccardi, entre otros) establecieron categorías de análisis que dan cuenta de la complejidad del tema: pobreza material y no material, activos sociales, pobreza de género, estrategias de supervivencia. Introdujeron, en el análisis, la dimensión sectorial (Ej.: Arriagada), familiar y territorial de la pobreza, donde las condiciones de vida de las personas y la exclusión son expresiones que sintetizan las múltiples situaciones de pobreza.

Una primera mirada a las estadísticas oficiales del Censo Nacional de Población y Vivienda 2001 reveló que una importante porción de los hogares de la región (15\%) estaban privados de satisfacer sus necesidades básicas e involucraba a un 18\% de la población total del recorte territorial estudiado. Estas diferencias porcentuales entre hogares y personas confirman que los hogares pobres contienen a un número mayor de personas que aquellos hogares que no tienen este tipo de carencia. También mostró una correlación entre crecimiento poblacional de los municipios y aumento de las NBI. En efecto, en aquellos donde mayor tasa de crecimiento poblacional hubo, como es el caso del partido de Florencio Varela, las condiciones de vida de sus habitantes desmejoraron; por otro lado, se trata de uno de los municipios que menor desarrollo industrial posee en la región estudiada y el que se encuentra ubicado más al sur del AMBA.

En contraposición con el caso anterior, el municipio de Avellaneda muestra mejores condiciones en sus indicadores, se trata de una zona con mayor grado de desarrollo industrial, con menor crecimiento poblacional y ubicada geográficamente al sur de la Ciudad Autónoma de Buenos Aires.

Una conclusión que se puede deducir de lo anterior es que, en el lapso analizado, las zonas urbanas que menos crecieron son aquellas donde hubo menores índice de NBI, mientras que las zonas que más crecieron fue donde se expandió el número de hogares y de habitantes con NBI.

Respecto de la evolución en torno a las NBI, se advirtió que tanto en la zona de estudio como en el resto de la provincia de Buenos Aires, durante los últimos veinte años, ha tenido una tendencia a la baja, reduciéndose hasta alcanzar niveles cercanos al 17,6\% de la población del conurbano. Durante el mismo período de estudio en el que las NBI muestran un rumbo hacia la baja, las LP y LI presentan un fuerte incremento. Esta aparente contradicción entre los indicadores responde a una suerte de espejismo estadístico dado por la definición de los indicadores: Es posible que un hogar no presente necesidades insatisfechas y que, al mismo tiempo, sea pobre. Básicamente, las NBI dan cuenta de indicadores de tipo estructural, vivienda, nivel de educación, hacinamiento, condiciones sanitarias, mientras que las LI y LP miden ingresos monetarios capaces de cubrir una canasta básica de alimentos y los gastos totales del hogar. El proceso que releva estos indicadores es la Encuesta Permanente de Hogares (EPH), que, durante el último decenio, ha tenido una trayectoria ascendente, hasta alcanzar niveles nunca vistos en la historia de la Argentina: en el 2002, en el conurbano bonaerense, el 64,4\% de las personas habitaban en hogares pobres, y el $30,5 \%$ pertenecían a hogares indigentes. El 
comportamiento del nivel de pobreza fue correlativo a la secuencia de los ciclos económicos de expansión y contracción, el nivel de desempleo, la distribución del ingreso y a la actividad de las pequeñas y medianas empresas, que son aquellas que mayor demanda de empleo tienen; sin embargo, hay que señalar que, en los momentos recesivos, la pobreza creció a una tasa que no pudo ser compensada totalmente por los descensos registrados en los ciclos de expansión. El resultado de este comportamiento diferencial fue una tendencia creciente y permanente a la extensión de la pobreza en umbrales cada vez más altos.

Es justamente en los períodos de alto desempleo (tasas cercanas al 25\%, en 2001) cuando se generan los niveles más altos de pobreza e indigencia.

Sin embargo, en la Región del AMBA, donde existe una mayor tasa de empleo respecto del total del país, existe una mayor presión de búsqueda de empleo, la tasa de desocupación es superior al promedio nacional y, al mismo tiempo, se registran las mayores tasas de subocupación horaria. Es importante destacar que, para las estadísticas oficiales, aquellas personas beneficiarias de los planes sociales son consideradas personas ocupadas. En general, la tasa del nivel de actividad tiende al crecimiento, y la tasa de desocupación, por oposición, tiene un comportamiento contrario y se reduce. Esta conducta podría estar asociada a la evolución favorable que están teniendo las pequeñas y medianas empresas en la zona. Otro dato que refuerza la tendencia anterior es la evolución del PBI, manteniéndose la elasticidad empleo-producto en 0,31.

Un problema de creciente importancia se refiere a la calidad de las ocupaciones que genera la economía. La crisis agravó la tendencia ya presente en los años noventa de precarización del empleo, es decir, de creación de puestos inestables, con bajos salarios y sin beneficios sociales. La situación social se completa con la estructura productiva localizada en el territorio en estudio, que se analiza en el apartado siguiente.

\section{LOS RASGOS PRODUCTIVOS DE LA SONA SUR DEL AMBA}

\section{La trama productiva: determinantes de localización y situación del entorno}

La configuración del mapa productivo urbano se va construyendo a través del tiempo como resultado de la confluencia de los soportes físicos y ambientales, entramado con el encadenamiento de las actividades y funciones de las relaciones sociales vigentes.

El mapa productivo urbano se despliega en una superficie que adquiere una dinámica y densidad cambiante, dependiendo de muchos factores, entre los más significativos está el rumbo y accionar político local y nacional, aunado a las tecnologías de producción y de los productos que circulan por los flujos de bienes y servicios que cruzan el territorio y se insertan en el mercado global.

En el área urbana que analizamos en este trabajo, habita la cuarta parte de la población del país, y es donde se produce una quinta parte de la riqueza nacional; por ello el espacio urbano que abarca solo el 0,1\% del territorio nacional adquiere una relevancia primordial desde el punto de vista del acontecer político y económico (Fidel, C; Di Tomaso, R; Farías, C (2005) Industria Manufacturera año 2004). ${ }^{1}$

Entre 1994 y 2004, se evidenció un fuerte proceso de destrucción industrial (-29.8\%), situación que fue compensada en los últimos años luego del proceso de devaluación en 2001, que volvió a encauzar a la economía argentina en el sendero de crecimiento económico, con generación de empleo y condiciones apropiadas para la germinación de nuevas pequeñas y medianas empresas, a partir del año 2002.

Las actividades industriales en el recorte territorial analizado dan cuenta de una estructura

${ }^{1}$ Los datos que aquí se presentan son el resultado de un proyecto conjunto de estudio de las PYME, en el que participaron la Universidad de Bologna, las Universidades Nacionales de Quilmes, la Matanza y San Martín, y el Ministerio de Economía de la Provincia de Buenos Aires. (Observatorio PyME regional. Conurbano Bonaerense, 2005). 
productiva donde predominan las referidas a la actividad alimenticia. La devaluación monetaria del año 2002 generó un contexto favorable para aumentar las exportaciones, un hecho que no tuvo un impacto muy favorable en la zona. En efecto, tan solo una pequeña porción (10\%) participa del mercado internacional, y el resto solo tiene posibilidades de competir con otros productores que operan en el mercado nacional.

Señalaremos algunas de las cuestiones que atraviesan la problemática productiva de la zona. Una de ellas es el desarrollo concentrado de manera desigual en algunos puntos del territorio.

Otro aspecto es que el impulso reciente de incremento de la producción de las PYME sigue el sendero que utiliza tecnologías relativamente poco innovadoras, y su producción se orienta, en su mayoría, a atender las demandas del mercado interno, con casi nula tendencia a ampliar las exportaciones.

Considerando la política gubernamental se debe apuntar lo mucho que hay por hacer. En especial, los gobiernos locales deben observar que el $73 \%$ de los empresarios manifestaron no mantener ningún contacto con los gobiernos municipales. Además, es ineludible advertir que, desde otras esferas del Gobierno, aprovechando los escenarios actualidad, se podrían diseñar e implementar políticas públicas de sostén y desarrollo local, para mejorar y ampliar la producción de las PYME, buscando que puedan afianzar y diversificar su producción, aplicando nuevas tecnologías que mejoren su capacidad instalada, su competitividad y la posibilidad de generar empleo estable y formal.

El entramado productivo no se trata de una distribución homogénea, sino que se concentra en algunos puntos del territorio, lo cual aumenta la brecha entre aquellos que poseen mayor capacidad de generación de empleo y aquellos que no. Estos territorios más sensibles a la evolución de la economía nacional se ubican espacialmente linderos a la Ciudad Autónoma de Buenos Aires, tuvieron un menor crecimiento poblacional entre censos $\mathrm{y}$, adicionalmente, menores tasas de población con NBI. De este modo, se pone de manifiesto la existencia de una fuerte correlación entre número de empresas PYME, tasa de desocupación y nivel de pobreza e indigencia, mostrando y consolidando así la importancia del estudio asociado con la evolución y desarrollo del sector PYME en la región. Estas zonas en las que existe mayor desarrollo industrial también muestran el grado de vulnerabilidad que poseen a los vaivenes del ciclo económico y que a la vez se encuentra vinculado a su perfil productivo. Algunos sectores industriales parecerían haber desarrollado mecanismos de resistencia a las tendencias desfavorables del ciclo.

\section{PRINCIPALES ACTIVIDADES INDUSTRIALES DEL CONURBANOSUR}

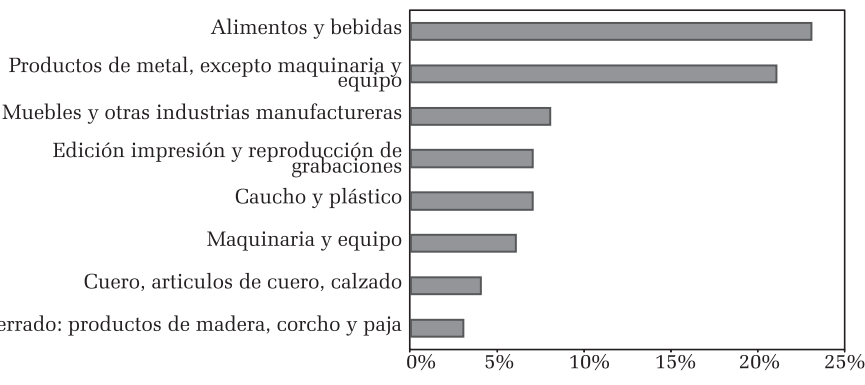

Las actividades industriales que concentran el mayor número de locales industriales de la zona de estudio corresponden a la actividad dedicada a la fabricación de alimentos y bebidas, seguida por la industria de productos de metal, excepto maquinaria y equipo. Como se puede observar en el cuadro anterior, el resto de las actividades se distribuye en diversos rubros industriales. De lo anterior surge que aquellas ramas productivas que demandan un mayor grado de tecnología tienen poco peso en el territorio, por lo que un estudio más específico sobre los sectores líderes sería de vital importancia para poder detectar la existencia de eslabonamientos productivos intra y extrasectoriales, y repensar así el diseño de políti- 
cas públicas dirigidas a fomentar la actividad de las PYME, que permitan iniciar el proceso de transformación que las ubique en el sendero de la competitividad y eficiencia, teniendo como horizonte su inserción en los circuitos del mercado internacional de bienes y servicios, principalmente en los mercados regionales, que contribuyan al desarrollo local de estos territorios atravesados por núcleos importantes de amplios sectores populares que viven en la pobreza y la exclusión .

Los ejes de análisis que se desarrollan en los próximos párrafos son los que hacen referencia al entorno urbano, definido a los fines de este trabajo como: accesibilidad, seguridad, contaminación ambiental, cargas impositivas locales, cortes de energía eléctrica.

Estos soportes materiales también son importantes en el desarrollo y normal desenvolvimiento de las firmas.

Una mirada hacia los indicadores que muestran las posibilidades de acceso a la planta arrojó que la décima parte de los responsables de los locales industriales lo consideró malo. Estas opiniones son muy heterogéneas si se tiene en cuenta la rama de actividad que desarrollan las firmas. En 2004, un poco menos de la décima parte de los responsables de los locales totales consideró que el nivel de accesibilidad al territorio era malo. No obstante, si se los observa según la actividad que desarrollan, se presentan valores muy heterogéneos, siendo los que corresponden a "mueblerías, productos de madera y otras actividades” los más perjudicados. Teniendo en cuenta el tamaño de cada empresa, aquellas con menor número de trabajadores (entre 10 y 200 empleados) son las que peores condiciones de accesibilidad presentan.

Otro ángulo del análisis empírico se refiere a los locales industriales que fueron víctimas de episodios delictivos. Los resultados mostraron que uno de cada cuatro locales industriales los tuvo. En este sentido, es importante resaltar que las firmas de menor tamaño fueron las más vulnerables, debido a su dificultad para destinar recursos a la contratación de seguridad privada y a que se corresponden con los sectores industriales con menor peso en relación al territorio.

Otro de los temas indagados por este trabajo se refiere a la contaminación ambiental.

En el estudio que realizamos para el Observatorio PYME del Conurbano Bonaerense Subregión Sur, se estimó un mayor porcentaje de locales pertenecientes a empresas con menos de diez ocupados, que piensan que soportan un nivel de contaminación elevado. Parecería, entonces, que existe una mayor conciencia de conservación del medio ambiente entre las empresas de menor tamaño. Al realizar el análisis separando cada actividad, se puede percibir que la de "alimentos y bebidas" es la que tiene un porcentaje significativamente mayor de locales que consideran convivir en un ambiente con alta contaminación. En el rubro alimenticio, se pueden encontrar sectores como los frigoríficos, una de las actividades productivas con altos grados de contaminación. Resulta llamativa la posición adoptada respecto del tema de actividades productivas como: "Sustancias y productos químicos"; "Caucho y plástico" o "Metales comunes, productos de metal, excepto maquinaria y equipo", que, siendo procesos productivos altamente contaminantes, no se reconocen como tales.

Por otro lado, la opinión acerca del elevado peso de los impuestos municipales se acentúa en el caso de los locales dedicados a las actividades "alimentos y bebidas". Este dato resulta de cierto interés, ya que se trata del sector más dinámico de la economía local.

Otra de las variables analizadas en el estudio del entorno urbano de las firmas se refiere a los cortes de energía no programados, que impactan de modo negativo en la actividad industrial. En este sentido, la mitad de locales de la zona estudiada tuvieron, por lo menos, dos cortes en el año sin previo aviso. Es importante destacar que los servicios de electricidad fueron privatizados en la Argentina durante los años noventa, y que las firmas distribuidoras se comprometieron a realizar inversiones en infraestructura que no se cumplieron. Consideramos, entonces, que si el ritmo de crecimiento de la economía se sigue sosteniendo en el tiempo, la situación podría llegar a transformarse en una crisis 
energética nacional y actuar como un freno para dicho crecimiento económico.

A modo de conclusión, se puede afirmar que las condiciones de localización alternan características que resultan satisfactorias con otras que no lo son tanto. Dentro de las primeras, puede mencionarse a los locales industriales, que consideran que soportan un alto nivel de contaminación ambiental, así como que el nivel de accesibilidad al territorio donde desarrollan su actividad es malo, y el alto porcentaje de locales que sufrieron episodios delictivos y que soportaron dos o más cortes de luz desde comienzos de 2004. Todo esto enmarcado en un contexto en el que se cree que los impuestos municipales son elevados.

A pesar de estas falencias, es bajo el porcentaje de locales que considera mudar el local. Una posible causa es que no han podido encontrar, dentro del conurbano, un lugar sin este tipo de fallas.

\section{Tecnología y complejidad organizativa}

En este apartado, indagaremos acerca del porcentaje de locales con procesos - o parte de ellos - automatizados, desde dos ejes centrales de análisis: por un lado, los referidos a las diferentes actividades que se desarrollan dentro de los locales $y$, por el otro, teniendo en cuenta los diferentes tamaños de las empresas. Los sectores con mayor número de locales, con todos o algunos de sus procesos automatizados, correspondieron al rubro "Maquinarias y aparatos eléctricos". En el otro extremo, se ubicaron las actividades "alimentos y bebidas". Estos resultados estarían mostrando la baja capacidad que posee el sector que lidera la producción en la zona de estudio para elaborar productos que posean las características tecnológicas necesarias para insertarse en los flujos de los mercados internacionales y regionales. Esta tendencia también fue observada en aquellos locales industriales de pequeño tamaño, con menos de diez ocupados.

Otra de las variables introducidas en el análisis se refiere a los locales industriales con certificaciones de calidad - normas ISO (International Organization for Standardization). Nuevamente aparece el sector de "alimentos y bebidas" como uno de los sectores industriales con menor número de locales manufactureros que poseen certificaciones de este tipo.

Parecería, entonces, que se comprueba la existencia de una fuerte asociación entre las variables: automatización, certificaciones de calidad y exportaciones.

En la zona sur del AMBA, uno de cada cinco locales exportó, durante el año 2002 y 2003, y dentro de los que participan del proceso exportador, la mayoría declaró formar parte de un grupo económico.

A modo de conclusión, podríamos subrayar: la automatización de procesos, o de parte de ellos, está difundida en más de la mitad de los locales. Por otro lado, un porcentaje bastante menor dispone de manual de procedimientos, y otro todavía más pequeño cuenta con certificaciones ISO. También un número muy pequeño de firmas forma parte de un grupo económico.

\section{Expectativas inmediatas}

Luego de la devaluación de la moneda en 2002, se presentó, en la Argentina, un contexto mucho más propicio para el desarrollo de las empresas en general, y de las pequeñas y medianas en particular. El nuevo tipo de cambio facilitó la exportación de productos, pero principalmente eliminó gran parte de la competencia externa que debieron soportar las empresas nacionales en el mercado interno. Este nuevo contexto se vio reflejado en la gran proporción de empresas que registraron un crecimiento y que tuvieron mayores montos de ventas en el año 2003 con respecto al 2002. No obstante, la recuperación no terminó aquí. La expectativa para el trienio 2004-2006 era, para la mayoría de las firmas, seguir en un sendero de crecimiento y colocar en el mercado montos de ventas mayores en el año 2004 con relación al 2003. 
A pesar de la recuperación, la cantidad de locales que realizaron inversiones durante el año 2003 continuó siendo baja, aunque más de la mitad de estos invirtieron más en dicho año que en el 2002. Además, las expectativas de inversión para el año 2004 fueron mayores para el 40\% de los locales. La principal falencia y, a la vez, el desafío más importante se relacionan con el acceso al mercado externo. Poco menos de un cuarto de los locales exportaron directamente en el año 2003, mientras que es muy baja la proporción de locales que realizaron un mayor porcentaje de ventas al exterior con respecto de la producción total, en comparación a 2002.

Dentro de este contexto de incrementos en los montos de las ventas, se observan actividades (encabezadas por "alimentos y bebidas") en las que son menos de la mitad las empresas que vendieron mayores montos en 2003 con respecto al año anterior. Es posible que las ventas de 2003 hayan sido un muy fuerte condicionante para las expectativas de ventas del año siguiente. Sin embargo, no en todas las actividades se vio una relación estrecha, y dicha proporción disminuyó para las expectativas de 2004.

Una relación similar se esperó en cuanto al crecimiento de las empresas. Pero nuevamente las observaciones mostraron que tales afirmaciones fueron, al menos, apresuradas.

En términos generales, las expectativas de crecimiento fueron mayores para el período 20042006, en comparación a las tasas de crecimiento de los años 2002-2003, por lo que puede suponerse que hay factores no considerados que hacen esperar un mayor crecimiento futuro que el experimentado en los años anteriores.

Con respecto a los valores de las inversiones, dos de cada tres empresas dedicadas a la producción de "Maquinaria y aparatos eléctricos" invirtieron durante el año 2003. Asimismo, más del $80 \%$ de estas empresas realizaron mayores inversiones en dicho año que en oportunidades anteriores. Entre las actividades que poseen un menor porcentaje de locales que invirtieron en 2003, se encuentran “alimentos y bebidas", lo cual podría mostrar una relación entre los niveles de exportaciones y los niveles de inversiones. A pesar de ello, la producción total exportada entre los años 2002 y 2003 presentó valores que no denotan un crecimiento importante.

\section{Inserción y funciones de la fuerza de trabajo activa}

En términos generales, pudo observarse que, a fines 2003, predominaron los locales con una cantidad media de personal, es decir, entre ocho y cuarenta trabajadores. A su vez, la gran mayoría empleaba asalariados con, por lo menos, estudios secundarios completos, mientras que eran muy pocos los que contrataban personal sin escolaridad.

Al contrario de lo que podría esperarse, no hay una relación lineal dentro de una misma actividad entre un alto porcentaje de locales que emplean asalariados con al menos estudios secundarios completos y un bajo porcentaje que contratan ocupados sin escolaridad, y viceversa. Es el caso de la actividad "papel, productos de papel, edición, impresión y reproducción de grabados", cuya proporción de locales en los que trabajan individuos con doce o más años de escolaridad es alta, al mismo tiempo que la cantidad relativa de locales que cuentan con asalariados sin escolaridad es significativamente mayor a la media de la zona.

De los enunciados anteriores podemos abordar algunas consideraciones relevantes a los fines específicos del estudio. En primer lugar, debemos señalar que los sectores más desposeídos de la población, en muchos casos, comparten el espacio urbano con la trama industrial de la zona. Este hecho contribuye a sumar penurias a la población, en el sentido de que los pobladores deben convivir con los efectos negativos que generan muchas de las actividades industriales en términos de contaminación ambiental, sobre todo las industrias que tienen actividades relacionadas con la producción de algunos alimentos y productos del 
cuero, tales como los frigoríficos, elaboración de chacinados, encurtidos y curtiembres, que depositan sus desechos en arroyos linderos a muchos asentamientos precarios y causan olores nauseabundos, emanación de productos contaminantes, ruidos molestos, entre otras externalidades negativas provocadas por la actividad industrial que tienen impacto sobre la salud, con escasos o ineficientes controles por parte de las autoridades locales para la preservación del medioambiente. De esta forma, los sectores más pobres soportan la carga negativa y contaminante de la producción de bienes, y en su mayoría se encuentran privados y excluidos del consumo.

Del mismo modo, quedó plasmado, en el análisis del entramado PYME, la vulnerabilidad de estas empresas con relación a las de mayor tamaño o grupos económicos nacionales e internacionales, respecto de las oscilaciones negativas en la evolución de las variables claves del funcionamiento de la macroeconomía nacional. En este sentido, las pequeñas y medianas empresas quedan atadas a los vaivenes de la economía en general y, por ende, el mercado de trabajo no se asienta sobre bases sólidas para la generación de empleo, que se encuentra directamente relacionada con la pobreza y ocasiona la falta de ingresos.

Por otro lado, se pudo observar que las PYMES poseen baja capacidad de incorporar tecnologías innovadoras y normas de calidad internacional que les permitan despegar de la evolución de la demanda de mercado un interno pequeño e insertarse en los flujos de mercado global, regional e internacional, con la elaboración de productos de mayor valor agregado.

Del presente estudio deriva que, para ello, es necesario realizar inversiones en tecnología de procesos, así como que, en su gran mayoría, los locales industriales realizan sus inversiones con fondos propios, poseen escasa o nula comunicación con las áreas productivas de los gobiernos locales, provinciales o nacionales y universidades públicas existentes en el territorio.

Así, queda evidenciada una potente desarticulación entre los diversos actores locales que conviven en el territorio de estudio y el despilfarro de recursos materiales y simbólicos que podrían contribuir a un mejoramiento en las condiciones de vida material y social de la población del recorte territorial estudiado.

\section{CONCLUSIONES E IDEAS A MODO DE PROPUESTAS}

En la exposición del trabajo, se puede advertir que, a nivel territorial, se registra una conexión relativamente directa entre mayor pobreza - exclusión y menor instalación de plantas productivas; mientras que es relativamente menor la pobreza - exclusión en las zonas donde se encuentra más extendida la trama productiva. Dicho mapa territorial, marcado por grietas y surcos de desigualdad y distancia del entramado social, requiere de una acción pública y privada que tienda a homogeneizar el espacio social y económico, para ir superando las situaciones de "crisis" presentes y futuras de la existencia material y simbólica.

El modo y los tiempos que requiere la resolución de la actual crisis dependen de muchos y complejos factores internos y externos. Una de las claves para salir de la crisis depende de las estrategias de acción que se adopten, ya sean tanto las iniciativas que broten desde el interior de las bases profundas de la sociedad, como las respuestas que se diseñen desde los despojos que operan los dispositivos gubernamentales.

Desde una óptica "socialmente inclusiva”, las posibilidades de diseñar y concretar un nuevo paradigma deben transitar por un cauce que estreche el diseño y la aplicación de la política económica con la política social, asociando las potencialidades e iniciativas emergentes de la población en un solo esquema productivo que optimice el uso los recursos, distribuya equitativamente la riqueza e iguale las posibilidades de crear un universo de participación de la población, en un sendero social amplio que restablezca los vínculos entre el mundo del trabajo y el social. 
La perspectiva de las formas económicas asociativas y solidarias constituye la más completa y eficaz manera de generar el desarrollo de los activos sociales, ya que concita los esfuerzos, iniciativas, capacidades y recursos de los propios sectores afectados por la pobreza y la marginación, aumenta los niveles de participación ciudadana e incrementa los niveles de desarrollo social y humano, permitiendo, así, promover la inserción de distintos segmentos de organismos asociativos y, en general, solidificar los lazos de existencia de la población más pobre.

Las formas de economía solidaria necesitan, para su eficaz desarrollo, la inducción de un fuerte flujo de conocimientos tecnológicos y criterios básicos, para conseguir o ampliar el financiamiento necesario y mejorar la gestión, a fin de elevar sus niveles de eficiencia en el uso de los recursos y factores que emplean, y así obtener sustentabilidad productiva y financiera. Por eso resulta de vital importancia el apoyo, la orientación, el seguimiento y la evaluación de las acciones implementadas de parte de los organismos públicos, con el objetivo de generar un desarrollo viable y sostenible en el tiempo.

Una alternativa, ubicada dentro del paradigma de la expansión de los activos individuales y comunitarios de poblaciones marginadas, puede estar centrada en la generación y expansión del hábitat material, vinculado a otras iniciativas productivas de la población de base urbana y rural (vivienda, centros de educación, salud, recreación, redes de producción y distribución, y servicios artesanales o industriales, entre otros), como soporte articulador de las condiciones de vida material de la población de menores o nulos ingresos y como factor que incremente el acervo de las variadas formas del "saber hacer" simbólico y real, a nivel individual y social, que permita un desarrollo permanente a lo largo del tiempo.

Desde esta perspectiva, es necesario transformar y recrear el Estado para que funcione como articulador y genere la promoción o expansión del financiamiento de distintas asociaciones solidarias del entramado de la sociedad, con el propósito de idear, de manera colectiva y participativa, un esquema de desarrollo sustentado a partir de un mayor financiamiento de pequeños proyectos, y se incremente así el nivel de participación comunitaria, contemplando los siguientes objetivos:

- Estimular la mejora y el aumento de la calidad y eficacia de las asociaciones relacionadas con los emprendimientos asentados en los sectores más pobres de la sociedad local.

- Impulsar la conformación, desde las propias organizaciones comunitarias, de nuevos circuitos de producción y circulación de bienes y servicios destinados a la construcción y el mantenimiento de los soportes productivos y el progreso social y económico de la población.

- Incrementar la producción eslabonada de insumos y productos apropiados para la reproducción social, simbólica y material. Estos deben ser prioritariamente de origen local y competitivo, para que puedan exportarse a otros países, en especial a los mercados de producción del MERCOSUR.

- Implementar medidas para volver a ocupar productivamente los distintos marcos construidos que están sin uso y localizados en el territorio, a través de la apertura de canales que se diseñen con estrategias operativas y financieras adecuadas a los requerimientos de cada zona.

- Diseñar y difundir medidas de relanzamiento de la producción de bienes y servicios artesanales y fabriles, que estructuren de forma sólida el mercado interno e inserten la producción local en las principales corrientes de intercambio vigentes en el mundo.

Para concretar los aspectos anteriores se deben utilizar al máximo las capacidades instaladas ociosas de los activos financieros, humanos y sociales, para diseñar e impulsar la generación de ventajas dinámicas de la producción nacional. Para ello, se debe avanzar en el cambio y la actualización del paradigma cultural, científico y tecnológico que sirva de sostén, y del despliegue del volumen y las potencialidades de las capacidades instaladas en nuestro país. 
La intervención, para ser viable y efectiva, deberá estar dirigida a generar un fuerte y penetrante impacto reconstituyente de la estructura de los pequeños y medianos emprendimientos artesanales y manufactureros en el territorio, acompañada de la ampliación de formas participativas y solidarias de organización que exterioricen los núcleos sociales que aún permanecen vivos en las profundidades de nuestra sociedad.

(Recebido para publicação em setembro 2009)

(Aceito em abril 2010)

\section{BIBLIOGRAFÍA}

ABRAMOVICH, A. L. y A. M. FEDERICO-SABATÉ. Condiciones económicas que justifican el impulso de una estrategia de desarrollo de economía social o del trabajo. Mundo Urbano (publicación electrónica) n. 18, 2002.

ALBURQUERQUE LLORENS, Francisco, et al. Revolución tecnológica y reestructuración productiva: impactos y desafíos territoriales. Buenos Aires: ILPES-PUC-Grupo Editor Latinoamericano, 1990.

ALTVATER, Elmar. De las realidades posibles; impedimentos en el camino del desarrollo, incluido. En: THIEL, Robert E. Arocena, D. Los ejes centrales del desarrollo local en Argentina. Buenos Aires: FLACSO, 2002.

BARBA SOLANO, Carlos (Comp.) Retos para la integración social de los pobres en América Latina. Rasgos de las insuficiencias urbanas y habitacionales en el Partido de Quilmes, Argentina. Buenos Aires: Ed. CLACSO - CROP, 2009.

BARRERA, Carlos A.; TORRES H. Ambiente: apropiación de la renta y comercio internacional. En: MINSBURG N.; VALLE H. W., El impacto de la globalización. Buenos Aires: Ediciones Letra Buena, 1994.

BECK, U. La sociedad del riesgo. Barcelona: Editorial Paidós, 1998.

BOISIER, Sergio. El vuelo de una cometa. Una metáfora para una teoría del desarrollo territorial. Revista Eure, n. 69, Santiago de Chile, 1997.

BORJA, Jordi Y CASTELLS, M. Local y global. La gestión de ciudades en la era de la información. Madrid: Taurus, 1998. competitividad de las empresas. El rol de las instituciones en el espacio global. Madrid: Miño y Dávila editoresUNGS, 2000

BOURDIEU, P. El sentido práctico. Madrid: Editorial Taurus, 1991.

BRESSE PEREIRA, C.A.; CUNILL GRAU, N. Lo público no estatal en la reforma del Estado. Buenos Aires: PaidósCLAD, 1998.

BURIN, D.; HERAS, A. I. Desarrollo local. Una respuesta a escala humana a la globalización. Buenos Aires: Ed. Ciccus/La Crujía, 2003.
CASTELLS, Manuel. La ciudad informacional. Madrid: Alianza, 1995.

CORAGGIO, J. L. Economía urbana. La perspectiva popular. Ecuador: Editorial Propuestas, 1998.

La propuesta de economía solidaria frente a la economía neoliberal. Buenos Aires: Mimeo, 2000.

Contribuciones posibles de la economía popular urbana a la transformación productiva con equidad. Instituto Fronesis: Quito, 1996.

La agenda del desarrollo local. Seminario de Desarrollo local, democracia y ciudadanía. Montevideo, 1996.

Descentralización, el día después... Cuadernos de Posgrado, Serie cursos y conferencias de la UBA. Buenos Aires, 1997

CORIAT, B. El Taller y el Robot. Ensayos sobre el fordismo y la producción en masa en la era de la electrónica. Buenos Aires: Siglo XXI Editores, 1992.

CUENYA, B.; FIDEL, C.; HERZER, H.; (Coord.) Fragmentos Sociales. Problemas urbanos de la Argentina. Buenos Aires: Siglo XXI Editores, 2004.

DE MATTOS, C. A. La descentralización ¿Una nueva panacea para enfrentar el subdesarrollo regional? En: LAURELLI, E.; ROFMAN, A. (Comps.), Descentralización del Estado. Buenos Aires: Ediciones CEUR, 1989

Evolución de las teorías del crecimiento. Instituto de Estudios Urbanos de la Pontificia Universidad Católica de Chile. Santiago de Chile, 1997.

Santiago de Chile, globalización y expansión metropolitana: lo que existía sigue existiendo. Revista EURE, n. 76, Santiago de Chile.

ELGUE, Mario C. Globalización, desarrollo local y redes asociativas, Buenos Aires: Corregidor, 1999.

FEDERICO-SABATÉ, A. M. Economía y sociedad de la Región Metropolitana de Buenos Aires en el contexto de la reestructuración de los 90, Buenos Aires: Al MargenUNGS, 2002

; VÁZQUEZ, G. Aportes a la economía metropolitana comparada, UNQ Argirópolis, Mundo Urbano, $\mathrm{n}$ 18, Buenos Aires, 2002.

FIDEL, C. Ladrillos más ladrillos menos. El comportamiento y las políticas de construcción en la Argentina, Buenos Aires, UNQ - Lecturas de Globalización y Economía Urbana, 1991.

et al. La política social en la Argentina en los noventa: el lugar del hábitat [inédito].

et al. Rasgos de las insuficiencias urbanas y habitacionales en el Partido de Quilmes, Argentina [publicación en proceso].

et al. Observatorio Pymes Regional. Conurbano Bonarense. UNM, 2005

et al. Industria Manufacturera año 2007 Observatorio Pyme, Regional Conurbano Bonaerense. UNM, 2007.

et al. Territorio, Condiciones de vida y Exclusión. El Partido de Quilmes, provincia de Buenos Aires, Argentina, Buenos Aires, CLACSO-CROP, 2008.

HARVEY, David. Globalización y urbanización, Revista Geographikós, n. 8, Año 7, Buenos Aires, 1997.

INDEC, Censo Nacional de población y vivienda 1991. Resultados definitivos. Partidos del Gran Buenos Aires por "localidad". Buenos Aires, Ministerio de Economía y Obras y Servicios Públicos, 1996. 
LUNGO, M. La tierra urbana, San Salvador: UCA Editores, 2000 .

MARSHALL, A. Contrataciones flexibles o trabajo precario. El empleo temporario y a tiempo parcial. En: GALIN: NOVICK (Comps.) La precarización del empleo en la Argentina. Buenos Aires: CEAL/CIAT/CLACSO, 1990.

MARSHALL, A. Consecuencias económicas de los regímenes de protección en América Latina. Revista Internacional del Trabajo, v. 113, n. 1. OIT, Ginebra, 1994.

MARTÍNEZ, D. Políticas de mercado de trabajo en la OCDE y en América Latina. Doc./Tbj. n. 42, Oficina Regional de OIT, Lima, 1996.

MATO DÍAZ, F. J. Estudio sobre las experiencias europeas con los instrumentos de evaluación de las políticas del mercado de trabajo, Serie Macroeconomía del desarrollo, Santiago de Chile, CEPAL, 2003.

MINISTERIO DE TRABAJO, EMPLEO Y SEGURIDAD SOCIAL. Gobierno Nacional de la República Argentina: http://www.trabajo.gov.ar/. Acceso: 20-05-05:

MONZA, A. Los dilemas de las políticas de empleo en la coyuntura argentina actual, Buenos Aires, Fundación OSDE/CIEP, 2002.

MONZA, A. y GIACOMETTI, C. Los Beneficiarios del Plan Jefas y Jefes de Hogar, Doc. de Trabajo 1. Versión preliminar. OIT, Buenos Aires, 2003.

NOCHTEFF, H. La economía argentina a fin de siglo: fragmentación presente y desarrollo ausente, Buenos Aires, EUDEBA/FLACSO, 1998.

OSUNA LLANEZA, J. L. La evaluación de las políticas de empleo: hacer de la necesidad virtud, VII Congreso Internacional del CLAD sobre la Reforma del Estado y de la Administración Pública, Lisboa, Portugal, 2002
PAUTASSI, L. et al. ¿Derecho social o beneficio sin derecho? El caso del Plan Jefas y Jefes. $6 .{ }^{\circ}$ Congreso de ASET, Buenos Aires, 2003.

RAPOPORT, M. Historia económica, política v social de la Argentina [1880-2000], Buenos Aires, Macchi, 2000.

RIQUELME G. y HERGER, N. Crisis y recesión económica: la búsqueda de nuevos sentidos para la educación y formación de los trabajadores. El caso argentino, IV Congreso ALAST, La Habana, 2003.

ROCA, E. Metamorfosis del empleo en Argentina. Diagnóstico, políticas y perspectivas, Buenos Aires, UBA, 2001. et al. Plan Jefas y Jefes de Hogar Desocupados: ¿Política de empleo o Política Social?, 6. ${ }^{\circ}$ Congreso de ASET, Buenos Aires, 2003.

ROFMAN, Alejandro. Las economías regionales a fines del siglo XX, Buenos Aires, Ariel, 1999.

Convertibilidad y desocupación en la Argentina de $\operatorname{los} 90$. Colección CEA-CBC. Buenos Aires, UBA, 1997.

SIEMPRO, Análisis del gasto social de la Administración Pública Nacional. En Informes de políticas y programas sociales, $\mathrm{N}^{\circ}$ 1, Buenos Aires, 2000.

Deuda social, Consejo Nacional de Coordinación de Políticas Sociales, Buenos Aires, Mimeo, 2003.

ZICCARDI, A. (Coord.) Pobreza, desigualdad social y ciudadanía: Los límites de las políticas sociales en América Latina, Buenos Aires, CLACSO-FLACSO-MéxicoIISUNAM, 2001.

Políticas sociales y gobiernos locales en el federalismo, IIS-UNAM, Argirópolis, Mundo Urbano, N. ${ }^{\circ} 18$, Buenos Aires, 2002 


\section{APPROXIMATION TO THE RELATIONS BETWEEN EXCLUSION, PRODUCTION AND TERRITORY}

\author{
Carlos Fidel, Raúl Di Tomaso, Cristina Farias
}

To approach the problematic of production, crossed with social and economic aspects, in the territory of the Metropolitan Area of the City of Buenos Aires (in Spanish, ZMCBA)

Methodology: The methodological way goes through the contextual presentation, continues in an analytical way, in territorial and thematic terms. The language develops itself around two main concepts: Production and Poverty. Both notions open broad and distinct lines for reflection and interpretation, most more or less compared. We will present a perspective of both "concepts" and will examine the possible relations that may be established between them. It ends with a series of suggestions that may serve as a trigger of new works or of possible concrete actions to better the lives of the inhabitants of the poorer areas.

KEYWORDS: production, poverty, exclusion, quality of life, territory.

\section{UNE APPROCHE DES ENSEMBLES EXISTANTS ENTRE EXCLUSION, PRODUCTION ET TERRITOIRE}

\section{Carlos Fidel, Raúl Di Tomaso, Cristina Farias}

Traiter de la problématique productive à partir de croisements de données concernant les aspects socioéconomiques dans la région métropolitaine de la ville de Buenos Aires (ZMCBA).

Méthodologie: Le parcours méthodologique va de la présentation du contexte à une analyse continue pour en arriver aux territoires et aux thématiques. Le langage tourne autour de deux concepts fondamentaux: la Production et la Pauvreté. Ces deux notions permettent une ouverture ample et distincte sur des axes de réflexions et d'interprétations qui, pour beaucoup, se confrontent. Nous fournirons un aperçu général concernant ces deux "concepts" et nous examinerons les liens possibles qui peuvent être établis entre eux. Nous terminerons par une série de propositions qui pourront servir de déclencheur à de nouveaux travaux ou à des actions possibles pour contribuer à l’amélioration de la vie des plus pauvres.

MoTS-CLÉs: production, pauvreté, exclusion, qualité de vie, territoire.

Carlos Hugo Fidel - Professor titular da Faculdade de Ciências Sociais da Universidad Nacional de Quilmes e Diretor Institucional do Programa - Observatório PyMES Zona Sul do Projeto de pesquisa Produção, Qualidade de Vida e Exclusão - UNQ. É Mestre e Doutor em Economia pela Universidad Nacional Autónoma de México - UNAM. Licenciou-se em Economia na UNS, República Argentina. É diretor da Revista de Ciencias Sociales (Segunda época) da UNQ e organizador, coordenador de edição e membro do Conselho Acadêmico da Revista Virtual de Investigação Urbana- Mundo Urbano. É Autor e co-autor de inúmeras publicações na área de estudos urbanos, pobreza e produção. Entre os seus livros destaca-se Fragmentos Sociales Problemas Urbanos de la Argentina

Raúl Di Tomaso - Sociólogo pela Universidad de Buenos Aires, com Mestrado em Análisis de la Opinión Pública pela Universidad Nacional de San Martín e em Generación y Análisis de Información Estadística pela Universidad Nacional de Tres Febrero (Argentina). É Docente pesquisador com dedicação exclusiva, investigador do Observatorio PyME. Foi consultor do Programa das Nações Unidas para o Desenvolvimento e da Organização Panamericana da Saúde nas temáticas de trabalho e da pobreza. É coautor de livros e artigos em revistas reconhecidas, tendo publicado em co-autoria com C. Fidel e Cristina Farias Território, Condiciones de Vida y Exclusión (CLACSO, 2008) e "Rasgos de las Insuficiencias Urbanas y Habitacionales en el Partido de Quilmes”. In: BARBA, Carlos. (Org.) Retos para la integración social de los pobres en América Latina. (CLACSO, 2009).

Cristina Farias - Graduada em Comércio Internacional pela Universidad Nacional de Quilmes (Ar.). Realizou diversos estudos posgraduados sobre Economia Urbana (Universidad Torcuato Di Tella) e sobre avaliação de projetos de investimentos na Universidad Nacional de Quilmes - UNQ. Docente e pesquisadora com dedicação exclusiva nesta Universidade, onde desenvolve pesquisas relativas às relações entre pobreza e produção no meio urbano. É pesquisadora permanente do Observatório PyMES do Conurbano Bonaerense e coautora de livros e periódicos reconhecidos. Entre seus trabalhos mais recentes destacam-se: o livro Território, Condiciones de Vida y Exclusión (Buenos Aires: CLACSO, 2008), em co-autoria com Fidel e Di Tomaso; e "Rasgos de las Insuficiencias Urbanas y Habitacionales en el Partido de Quilmes". In: Barba, Carlos. Retos para la integración social de los pobres en América Latina (Buenos Aires: CLACSO, 2009). 